\title{
A PRIORI BOUNDS FOR TEMPERATURE IN CIRCULATING FUEL REACTORS*
}

\author{
BY \\ JOEL FRANKLIN AND HERBERT B. KELLER \\ Institute of Mathematical Sciences, New York University
}

1. Introduction. The neutron density, $u$, and temperature, $v$, in a circulating fuel reactor satisfy non-linear partial differential equations of the form

$$
\begin{gathered}
u_{t}=u_{x x}+A(v) u, \\
v_{t}+c v_{x}=u \quad(0 \leq x \leq a, t \geq 0) .
\end{gathered}
$$

These equations are in dimensionless form; $t$ is a time-variable; $x$ is a space-variable; and $c$ is the positive, constant speed at which the fuel flows through the reactor. $A(v)$ is a given function of $v$. The neutron density is zero at the boundaries of the reactor:

$$
u(0, t)=u(a, t)=0 .
$$

The input fuel is kept at a constant temperature, say

$$
v(0, t)=0,
$$

in an appropriately chosen scale. The initial state of the reactor is given by

$$
u(x, 0)=u_{0}(x), \quad v(x, 0)=v_{0}(x),
$$

where $u_{0}, v_{0}$ are known functions. A physical derivation of the above equations, with $A(v)=$ constant $-v$, appears in a forthcoming report by J. Fleck [5].

The purpose of this paper is to determine $a$ priori bounds for norms of $u$ and $v$. Under natural assumptions on the given functions $A, u_{0}, v_{0}$, we suppose that smooth solutions $u, v$ exist to the mixed initial- and boundary-value problem posed by the preceding equations. We then derive numerical bounds, depending only on the given functions, for the maximum-norm of $v$ and for an integral-norm of $u$. These bounds will hold in the infinite domain $0 \leq x \leq a, 0 \leq t<\infty$. Our results are found by the elementary sort of argument used by E. Hopf [1] and by L. Nirenberg [2] in their justifications for the maximum principles for elliptic and for parabolic equations.

To prove a preliminary result, we shall use the following form of the weak maximum principle for parabolic equations. Let $U(x, t)$ be a solution of the equation

$$
U_{t}=U_{x x}+\phi(x, t) U
$$

which is twice continuously differentiable in the closed rectangle

$$
R_{T}: \quad 0 \leq x \leq a, \quad 0 \leq t \leq T ;
$$

"Received April 1, 1955. This work was sponsored by the United States Atomic Energy Commission. The authors wish to acknowledge their indebtedness to J. Fleck of the Brookhaven National Laboratory, who suggested a specific problem from which the present work originates. 
let $\phi(x, t)$ be continuous and $\leq 0$ in $R_{T}$; assume that $U$ takes positive values in $R_{T}$; then $U$ attains its maximum value on one of the boundary segments

$$
x=0, \quad t=0, \quad x=a ;
$$

similarly, if $U$ takes negative values in $R_{T}$, then $U$ attains its minimum value on (1.7). This principle has long been known in various forms; see for example M. Picone [3]. In [2] Nirenberg proves the strong maximum principle, which asserts that, if $U$ attains a positive maximum or a negative minimum at any point of $R_{T}$ not in the set (1.7), then $U \equiv$ constant.

2. Assumptions on the given functions. We suppose that the given functions $u_{0}(x), v_{0}(x)$ are twice continuously differentiable* functions of $x$ satisfying the boundary conditions

$$
u_{0}(0)=u_{0}(a)=0, \quad v_{0}(0)=0 .
$$

Because $u$ represents a density, we shall require

$$
u_{0}(x) \geq 0 \quad(0 \leq x \leq a) .
$$

Let

$$
\alpha=\min v_{0}(x), \quad \beta=\max v_{0}(x) \quad(0 \leq x \leq a) .
$$

We suppose that $A(w)$ is a given, continuous function defined for all $w \geq \alpha$. Since $\alpha$ $\leq v_{0}(0)=0$, we may define

$$
B(w)=\int_{0}^{w} A(\omega) d \omega \quad(w \geq \alpha) .
$$

We now make the important assumption

$$
\limsup _{w \rightarrow \infty} B(w)<\min (\gamma, 0),
$$

where

$$
\gamma=\min \left[-u_{0}(x)+c v_{0}^{\prime}(x)+v_{0}^{\prime \prime}(x)+B\left(v_{0}(x)\right)\right] \quad(0 \leq x \leq a) .
$$

This assumption does not appear to be unnatural; for example, if $A(w) \equiv$ constant- $w$, we have $B(w) \rightarrow-\infty$ as $w \rightarrow \infty$, so that (2.5) holds regardless of the value of $\gamma$. Since $B(w)$ is a continuous function, with $B(0)=0$, assumption (2.5) implies that there exists a unique, finite, non-negative number $M$ such that

$$
B(M)=\min (\gamma, 0), \quad \text { and } \quad B(w)<\min (\gamma, 0) \quad \text { for } \quad w>M .
$$

\section{Boundedness of temperature.}

Theorem. Let $u(x, t), v(x, t)$ be defined for $0 \leq x \leq a, 0 \leq t<\infty$ as functions with continuous derivatives up to the third order. Let $u, v$ satisfy the differential equations (1.1) and the boundary and initial conditions (1.2), (1.3), (1.4). Let the given functions $u_{0}, v_{0}, A$ satisfy the conditions of Sec. 2 . Then $v(x, t)$ is bounded; $v$ satisfies the inequality

$$
\alpha \leq v(x, t) \leq \max (\beta, M),
$$

where $\alpha, \beta, M$ are the constants defined in (2.3) and (2.7).

*We will not attempt here or in the statement of the theorem to use the weakest possible assumptions of smoothness. 
Proof. As a preliminary result, we prove that $u(x, t)$ is $\geq 0$ for all $t \geq 0$. Consider the rectangle $R_{T}$ defined by (1.6). Following Nirenberg, we define

$$
\lambda=\lambda(T)=\max A[v(x, t)] \quad \text { for } \quad(x, t) \text { in } R_{T}
$$

and define

$$
U(x, t)=e^{-\lambda t} u(x, t) .
$$

Then $U$ satisfies Eq. (1.5) with

$$
\phi(x, t)=A[v(x, t)]-\lambda .
$$

Since $\phi$ is $\leq 0$ in $R_{T}$, we may conclude from the maximum principle that $U$, and therefore $u$, assume negative values in $R_{T}$ only if $U$ assumes a negative minimum on one of the segments (1.7). But $u$, and therefore $U$, are $\geq 0$ on (1.7) because of the boundary conditions (1.2) and the inequality (2.2). Therefore, $u$ is $\geq 0$ in $R_{T}$. Since $T$ is arbitrary, $u(x, t)$ is $\geq 0$ in $R_{\infty}$, i.e. for all $t \geq 0$.

To prove the theorem it suffices to show that $v(x, t)$ satisfies the inequalities (3.1) in every finite, closed rectangle $R_{T}$. We consider $R_{T}$ as the sum of four boundary segments and the interior, according to the following definitions:

$$
\begin{aligned}
N: & 0<x<a, \quad t=T ; \\
E: & x=a, \quad 0<t \leq T ; \\
S: & 0 \leq x \leq a, \quad t=0 ; \\
W: & x=0, \quad 0<t \leq T ; \\
I: & 0<x<a, \quad 0<t<T .
\end{aligned}
$$

We also define sets $R_{T}^{-}$and $R_{T}^{+}$as the intersections of $R_{T}$ with the half-planes $c t-x \leq 0$ and $c t-x>0$.

To find a lower bound for $v$, we draw from each point $p$ in $R_{T}$ the segment of positive slope $1 / c$ extending to a point $p^{\prime}$ on $S+W$. The point $p^{\prime}$ lies on $S$ or on $W$ according as the point $p$ lies in $R_{T}^{-}$or in $R_{T}^{+}$. Since $u \geq 0$, it follows by integration of the equation $v_{t}+c v_{x}=u$ that $v\left(p^{\prime}\right) \leq v(p)$. By (2.3) and (1.3) we have $v \geq \alpha$ on $S$ and $v=0$ on $W$. Therefore,

$$
v \geq \alpha \text { in } R_{T}^{-} \quad \text { and } \quad v \geq 0 \text { in } R_{T}^{+},
$$

from which, since $\alpha \leq v_{0}(0)=0$, we find the required lower bound

$$
v \geq \alpha \text { in } R_{T} .
$$

Using the definition (2.4) of $B$, we may eliminate $u$ from (1.1) to obtain the single equation for $v$

$$
\left(\frac{\partial}{\partial t}+c \frac{\partial}{\partial x}\right)\left[-v_{t}+v_{x x}+B(v)\right]=0 .
$$

This equation implies that there exists a continuous function $f$ for which

$$
v_{t}=v_{x x}+B(v)+f(c t-x) .
$$


To find an upper bound for $v$ we shall need an upper bound for $f$. On $S$ (3.11) becomes

$$
\begin{aligned}
f(-x) & =v_{t}(x, 0)-v_{x x}(x, 0)-B[v(x, 0)], \\
& =u_{0}(x)-c v_{0}^{\prime}(x)-v_{0}^{\prime \prime}(x)-B\left[v_{0}(x)\right] .
\end{aligned}
$$

Therefore, by the definition (2.6),

$$
f(y) \leq-\gamma \quad(-a \leq y \leq 0) .
$$

On $W(3.11)$ becomes

$$
f(c t)=v_{t}(0, t)-v_{x x}(0, t)-B[v(0, t)] .
$$

But $v=v_{t}=0$ on $W$, and $B(0)=0$. Since $u=0$ on $W$, the second equation (1.1) gives $v_{x}=0$ on $W$. But, by (3.8), $v(p) \geq 0$ in $R_{T}^{+}$, so that $v(p) \geq 0$ in a neighborhood of each point of $W$. Therefore,

$$
v_{x x} \geq 0 \text { on } W
$$

It now follows from (3.13) that

$$
f(y) \leq 0 \quad(y>0) .
$$

Combining (3.12) and (3.15), we find

$$
f(c t-x) \leq\left\{\begin{array}{rll}
-\gamma & \text { for } & (x, t) \text { in } R_{T}^{-} \\
0 & \text { for } & (x, t) \text { in } R_{T}^{+} .
\end{array}\right.
$$

Let $P$ be a point at which $v$ attains its maximum value in $R_{T}$. Then $P$ lies in exactly one of the four continua $W, S, N+I, E$. If $P \varepsilon W$, then $v(P)=0$, by the boundary condition (1.3). If $P \varepsilon S$, then $v(P)=\beta$, by (2.3).

Suppose $P \varepsilon N+I$. Since $v(P)$ is the maximum value of $v$, and since the set $N+I$ is open with respect to $x$ and open from below with respect to $t$, we must have

$$
v_{x}(P)=0, \quad v_{x x}(P) \leq 0, \quad v_{t}(P) \geq 0 .
$$

Therefore, by (3.11) and (3.16),

$$
B[v(P)] \geq-f \geq \min (\gamma, 0) .
$$

From (2.7) we now conclude

$$
v(P) \leq M .
$$

Finally, suppose $P \& E$. Since $v(P)$ is the maximum value of $v$, the definition (3.4) of $E$ leads to the inequalities

$$
v_{x}(P) \geq 0, \quad v_{t}(P) \geq 0 .
$$

But $v_{t}+c v_{x}=u=0$ on $E$, and $c$ is $>0$. Therefore, (3.20) yields

$$
v_{x}(P)=0, \quad v_{t}(P)=0 .
$$

From the maximum-property of $v(P)$ we may now conclude

$$
v_{x x}(P) \leq 0 .
$$


From (3.21) and (3.22) follow (3.17) and, hence, the inequality $v(P) \leq M$. Summarizing the results for all of the four sets $W, S, N+I$, and $E$, we find $v(P) \leq \max (\beta, M)$ regardless of where the maximum value $v(P)$ is attained in $R_{T}$. This completes the proof of the theorem.

4. Boundedness of a norm of neutron density. By comparing the equations

$$
u_{t}=u_{x x}+A(v) u, \quad u_{t}^{*}=u_{x x}^{*}+\lambda u^{*},
$$

where $\lambda$ is a constant $\geq A(v)$, one easily obtains (see Pólya and Szegö [4]) an inequality of the form

$$
u(x, t) \leq K \exp \left(\lambda-\pi^{2} / a^{2}\right) t,
$$

where $K$ is a positive constant. According to (3.1) we shall certainly have $\lambda \geq A(v)$ if we define

$$
\lambda=\max A(w) \quad[\alpha \leq w \leq \max (\beta, M)] .
$$

Then (4.1) shows that the non-negative function $u$ is bounded if the length $a$ is sufficiently small, i.e. if $a^{2} \leq \pi^{2} / \lambda$. But this restriction is too strong in cases of physical interest.

If $a$ is unrestricted, the arguments of Sec. 3 evidently do not yield an upper bound for the neutron density $u(x, t)$. However, as an immediate consequence of the theorem, we can find a bound for a certain integral-norm of $u$. Define the boundaries

$$
E_{\infty}: x=a, t>0 ; \quad S: 0 \leq x \leq a, t=0 ; \quad W_{\infty}: x=0, t>0 .
$$

From a point on $E_{\infty}$, with ordinate $t$, we draw the segment $L_{t}$ of slope $1 / c$ to a point on $S+W_{\infty}$. We then define

$$
n(t)=\int_{L_{t}} u d s
$$

this integral may be properly called a norm, since $u$ is $\geq 0$. By the second equation (1.1) we have

$$
\left(1+c^{2}\right)^{1 / 2} \frac{d v}{d s}=u \text { on } L_{t}
$$

Therefore,

$$
n(t)=\left(1+c^{2}\right)^{1 / 2}\left[v(a, t)-v\left(a^{\prime}, t^{\prime}\right)\right],
$$

where $\left(a^{\prime}, t^{\prime}\right)$ equals $(a-c t, 0)$ or $(0, t-a / c)$ according as $c t-a \leq 0$ or $c t-a>0$. Thus, by (2.3) and (1.3),

$$
-v\left(a^{\prime}, t^{\prime}\right)\left\{\begin{array}{lll}
\leq-\alpha & \text { if } & c t-a \leq 0 \\
=0 & \text { if } & c t-a>0 .
\end{array}\right.
$$

Since, by the theorem, $v(a, t) \leq \max (\beta, M)$, we have proved the following result:

Corollary. Let the conditions of the theorem be satisfied, and let the norm $n(t)$ be defined by (4.2). Then

$$
\left(1+c^{2}\right)^{-1 / 2} n(t) \leq\left\{\begin{array}{lll}
\max (\beta, M)-\alpha & \text { if } & c t-a \leq 0 \\
\max (\beta, M) & \text { if } & c t-a>0 .
\end{array}\right.
$$




\section{REFERENCES}

[1] E. Hopf, Elementare Bemerkungen über die Lossungen partieller Differentialgleichungen zweiter Ordnung vom elliptischen Typus, Sitzungsberichte der Preussischen Akademie der Wissenschaften, 19, 147-152 (1927)

[2] L. Nirenberg, A strong maximum principle for parabolic equations, Communs. on Pure and Appl. Math., 6, 167-177 (1953)

[3] M. Picone, Sul problema della propagazione del calore in un mezzo privo di frontiera, conduttore, isotroppo e omogeneo, Mathematische Annalen, 101, 701-712 (1929)

[4] G. Pólya and G. Szegö, Sur quelques proprietés qualitatives de la propagation de la chaleur, Compt. Rend., 192, 1340-1342 (1931)

[5] J. Fleck, Temperature-dependent kinetics of circulating fuel reactors, Brookhaven National Laboratory Report, in preparation 